\title{
Parcerias entre Universidades e Poder Judiciário: experiência de Minas Gerais
}

\author{
Partnerships between universities and Judiciary: Minas Gerais experience \\ Las asociaciones entre Universidad y Poder Judicial: la experiencia de Minas Gerais
}

\author{
José Luiz Nogueira ${ }^{1}$ \\ Lélia Carvalho ${ }^{2}$ \\ Luciana Dadalto ${ }^{3}$
}

\begin{abstract}
RESUMO: O presente artigo científico tem como objetivo apresentar a parceria entre o NATS do HC-UFMG com o Tribunal de Justiça de Minas Gerais para emissão de notas técnicas em processos que envolvam judicialização da saúde. Para tanto, apresenta-se um caso real, em que a nota técnica emitida demonstra a falta de evidência científica para a requisição do medicamento pretendido e a falta de entendimento do Poder Judiciário para decisões mais acertadas. Ao longo do desenvolvimento, demonstra-se os resultados e os desafios do trabalho do NATS, concluindo pela necessidade em reforçar e posteriormente rever o papel do NATS na judicialização da saúde.
\end{abstract}

Palavras-chave: Direito à saúde. Medicamentos. Ações Judiciais.

ABSTRACT: This research paper aims to present the partnership between NATS HC -UFMG and the Court of Justice of Minas Gerais to issue technical notes in processes involving health judicialization. Therefore, it presents a real case, in which the technical note issued demonstrates the lack of scientific evidence to the request of the desired product and the lack of understanding of the judiciary to better decisions. Throughout the development, it demonstrates the results and challenges of NATS's work, concluding with the need to strengthen and then review the role of NATS in health judicialization.

Keywords: Legal right to heath. Drugs. Judicial suits.

RESUMEN: Este trabajo de investigación tiene como objetivo presentar la asociación entre NATS HC -UFMG con el Tribunal de Justicia de Minas Gerais para emitir notas técnicas en los procesos que implican la legalización de la salud. Por lo tanto, se presenta un caso real, en que la nota técnica emitida demuestra la falta de evidencia científica a la solicitud del producto deseado y la falta de entendimiento del Poder Judicial a mejores decisiones. A lo largo del desarrollo, demuestra los resultados y desafíos del trabajo del NATS, concluyendo con la necesidad de fortalecer y luego revisar el papel del NATS en la judicialización de la salud.

Palavras-Ilave: Derecho a la salud. Medicamentos. Acciones judiciales.

\footnotetext{
${ }^{1}$ Médico Especialista em Clínica Médica pelo Hospital das Clínicas-UFMG. Médico do Hospital das Clínicas. Coordenador do Núcleo de Avaliação de Tecnologia em Saúde (NATS) do HC-UFMG. joseluizsnogueira@gmail.com

${ }^{2}$ Médica especialista em Pediatria. Mestre em Saúde da Criança e do Adolescente pela Faculdade de Medicina pela UFMG. Médica do NATS - HC-UFMG - EBSERH. E-mail: leliamac@gmail.com.

${ }^{3}$ Doutora em Ciências da Saúde pela faculdade de Medicina de Minas Gerais. Mestre em Direito Privado pela PUC-Minas. Advogada. Professora do Centro Universitário Newton Paiva e da Faculdade Batista. Email: luciana@dadaltoecarvalho.com.br
} 


\section{Introdução}

\section{Relato de Caso}

Trata-se de requisição de Nota Técnica (NT) pelo Tribunal de Justiça de Minas Gerais (TJMG), endereçada para o Núcleo de Avaliação em Tecnologia em Saúde do Hospital das Clínicas da Universidade Federal de Minas Gerais (NATS HC/UFMG), sobre a indicação dos medicamentos TRASTUZUMABE e PERTUZUMABE, para tratamento de paciente do sexo feminino, diagnosticada com adenocarcinoma de cólon metastático (CID-10 C18).

Em abril de 2013, foi submetida à retossigmoidectomia associada à ressecção da parede da bexiga, ovário e trompa esquerdos, devido abdome agudo perfurativo. Foi evidenciada metástase para o pulmão, linfonodos de cadeia mamária à esquerda e fígado. Iniciou quimioterapia sistêmica com FOLFIRI (flourouracil, leucovorin e irinotecano) em combinação com bevacizumabe, obtido judicialmente. Em setembro de 2013 foi submetida hepatectomia direita regrada + enucleação de nódulos em lobo esquerdo + linfadenectomia + fechamento da ileostomia. Em outubro de 2013 fez retirada das lesões metastáticas do pulmão. Em novembro de 2013 iniciou tratamento adjuvante com Xelox® (capecitabina). Entretanto, a paciente com um ciclo desenvolveu intolerância e o tratamento foi interrompido, dando início ao esquema quimioterápico FOLFOX (fluorouracil, leucovorin e oxaliplatina). Após quatro ciclos, os exames de imagem evidenciaram recidiva da doença em cólon ascendente.

Em fevereiro de 2014 foi reiniciada quimioterapia com FOLFIRI + Bevacizumabe, obtido judicialmente. Após 10 ciclos apresentou progressão clínica da doença e sintomas de toxicidade limitantes. Em novembro de 2014 foi iniciado o tratamento com regorafenibe, obtido judicialmente, que fez uso até março de 2015, quando foi detectada nova progressão da doença.

Em março de 2015 iniciou tratamento com trastuzumabe emtansina - Kadcyla® (terapia utilizada em câncer de mama), que foi obtido judicialmente e utilizado na paciente, pelo fato da mesma possuir marcador tumoral semelhante, (HER2+), conforme justificativa do médico assistente, mesmo sabendo que se tratava de tratamento experimental. A paciente realizou aplicações de trastuzumabe emtansina em março, abril, maio e junho de 2015. Entretanto, evoluiu com progressão tumoral em pulmão e cólon transverso. 
Foi então judicializado outro esquema: trastuzumabe + pertuzumabe + capecitabina (novamente esquema experimental) e solicitada avaliação do caso pelo NATS, que após analise técnica, verificou que os medicamentos Kadcyla ${ }^{\circledR}$ (trastuzumabe emtansina) e Perjeta $\AA^{(p e r t u z u m a b e) ~ f o r a m ~ i n d i c a d o s ~ f o r a ~ d e ~ b u l a, ~ p o i s ~ n a ̃ o ~ h a ́ ~ n e n h u m a ~ b a s e ~ c i e n t i ́ f i c a ~}$ que corrobore o fato de pacientes portadores de câncer colorretal com HER2-positivo, possam ser tratados com o mesmo esquema terapêutico de pacientes portadoras de câncer de mama com HER2-positivo. Trata-se de abordagem terapêutica absolutamente empírica e experimental, diante de um quadro grave, de prognóstico sombrio, de uma paciente submetida a várias intervenções cirúrgicas e pelo menos oito linhas de tratamento. Além do mais não há indicação de bula do uso do trastuzumabe emtansina, assim como o pertuzumabe para tratamento de câncer colorretal. O custo aproximado de toda medicação judicializada é aproximadamente $R \$ 1.000 .000,00$.

Assim, a conclusão foi pela não recomendação dos medicamentos para o tratamento proposto com base em total falta de evidências científicas (1). No entanto, o resultado do julgamento em segunda instância, por dois votos a um, foi pelo deferimento do medicamento apesar da inexistência de qualquer evidência e da demonstração que o tratamento proposto é experimental. Apesar de não haver cruzamento de dados entre as notas técnicas elaboradas pelo NATS e a o resultado do processo judicial, a maioria das decisões tem concordância com as recomendações do NATS.

\section{Contextualização}

O convênio entre NATS HC-UFMG, SES e TJMG foi firmado em 2012, como uma ação do TJMG à recomendação n. 31/2010 do CNJ (2). O objetivo é a produção de Notas Técnicas a partir da solicitação dos juízes. As justificativas para o convênio são:

Considerando o aumento exponencial das demandas judiciais que objetivam acesso insumos de saúde;

Considerando as dificuldades dos magistrados em formar convencimento alinhado com a realidade das demandas que envolvam questões de saúde, em razão da complexidade da matéria, instruídas as ações com relatórios em linguagem técnica;

Considerando que a concessão de liminar nesse tipo de feito acarreta, não raro, dispêndio desnecessário de cifras elevadas, com comprometimento do erário, por não disporem os magistrados de instrumentos próprios ao esclarecimento das questões relacionadas, com o uso dos quais poderiam dar solução adequada às demandas; 
Considerando a diversidade de alternativas de tratamento, em termos tecnológicos, que vêm sendo colocadas à disposição pela ANVISA, e a necessidade de conhecimento e avaliação delas para que se dê ao processo o desate mais justo, tanto para o requerente das medidas judiciais quanto para o Poder Público;

Considerando o disposto nas Recomendações no 31/2010 e nํ 36/2011, do Conselho Nacional de Justiça, por meio das quais aquele Órgão sugere aos Tribunais Estaduais e Federais, a celebração de convênios para disponibilização de apoio técnico aos magistrados na formação de seu convencimento quando da apreciação das questões clínicas apresentadas pelas partes nas ações relativas à saúde;

Considerando que esse apoio, para ser efetivo e ter eficácia, deve ser fornecido por profissionais capazes de prestar ao magistrado uma consultoria técnica que o oriente sobre os diversos aspectos e intercorrências que permeiam a questão ${ }^{4}$.

A estratégia para constituir a parceria, que desencadeou o convênio TJ/SES/NATS foi o financiamento realizado pelo Gestor Estadual, através de contrato entre SES e Fundação de apoio à Universidade para remuneração das atividades dos NATS e um termo de Cooperação entre TJ e SES, para dar legitimidade a todo processo. O NATS responde técnica e diretamente apenas ao Poder Judiciário.

Ressalte-se que o convênio só foi possível em virtude de parceria prévia do NATS HC-UFMG com o Grupo de Avaliação de Tecnologias (GATS) da UNIMED Belo Horizonte desde 2006, que já tinha experiência em elaboração de notas técnicas embasadas em medicina baseada em evidências $(\mathrm{MBE})^{5}$ (3). Juntas, portanto as duas instituições vêm de longa trajetória na discussão de avaliação de tecnologia em saúde (ATS), além do desenvolvimento de vários projetos junto ao Ministério da Saúde, Agencia Nacional de saúde, Organização Pan-Americana de Saúde (OPAS) e outros. Como é importante para o Tribunal de Justiça trabalhar com uma instituição pública, acadêmica e, sobretudo sem conflitos de interesses o NATS apresentava, em 2012, época da assinatura do contrato, condições técnicas e operacionais de responder às demandas do Poder Judiciário.

Essas parcerias pretéritas possibilitaram ao NATS a feitura e desenvolvimento do convênio com o TJMG pois, em 2012, o NATS tinha profissionais e know how suficientes

\footnotetext{
${ }^{4}$ Estes considerandos estão na minuta do termo de referência para definição dos requisitos técnicos e operacionais exigidos das empresas especializadas no fornecimento de notas técnicas, elaborada pela assessoria do TJMG, mas que nunca foi assinado em virtude dos entraves burocráticos citados nesse artigo.

5"MBE se traduz pela prática da medicina em um contexto em que a experiência clínica é integrada com a capacidade de analisar criticamente e aplicar de forma racional a informação científica de forma a melhorar a qualidade da assistência médica”. LOPES, A. A. Medicina Baseada em Evidências: a arte de aplicar o conhecimento científico na prática clínica. Rev Ass Med Brasil 2000; 46(3), p. 285.
} 
para elaborar notas técnicas para os processos judiciais, assim como a manutenção e eventual ampliação do convênio.

Ademais, desde o ano de 2015, o NATS HC-UFMG coordena um curso semestral de Pratica de Saúde Baseada em Evidências, para profissionais tanto do HC quanto de outras instituições públicas, com objetivo de aplicação dos conhecimentos na assistência, ensino e formação de profissionais capacitados em ATS que, eventualmente elaboram as notas técnicas emitidas pelo NATS.

\section{A Judicialização da Saúde e os NATS}

O artigo 196 da Constituição Federal dispõe que: "A saúde é direito de todos e dever do Estado, garantido mediante políticas sociais e econômicas que visem à redução do risco de doença e de outros agravos e ao acesso universal e igualitário às ações e serviços para sua promoção, proteção e recuperação" (4).

Para materializar o disposto nesse artigo foi criado o Sistema Único de Saúde (SUS), projeto que assume e consagra os princípios da Universalidade, Equidade e Integralidade da atenção à saúde da população (5).

Ocorre que a consolidação do SUS enfrenta dificuldades, próprias de uma sociedade com processo democrático recente, enormes déficits sociais e na qual vicejam distintas concepções acerca do tamanho e do papel do Estado e da Política de Seguridade Social. Há uma tensão permanente entre os avanços sociais alcançados e as barreiras e retrocessos impostos (5). Umas dessas barreiras é a chamada judicialização da saúde.

Iniciada em período mais recente, nos últimos dez anos muito atrelada a um processo geral de judicialização da sociedade brasileira, com consequências importantes na consolidação do SUS. Diversas são as causas atribuídas à judicialização da saúde, mas aqui optou-se por analisar cinco delas, assumindo, o caráter resumido dessa análise:

a) Interpretação extensiva do art.196 da Constituição Federal: A maior parte dos atores que protagoniza a judicialização da saúde utiliza apenas a primeira parte do artigo" a saúde é direito de todos e dever do Estado" para justificar uma suposta obrigação estatal de prover tudo o que se refere à saúde aos cidadãos brasileiros. Estes atores, escondidos na justificativa de estarem aplicando preceito constitucional, interpretando-o de forma absurdamente extensiva, desconsideram as consequências desse suposto dever do Estado de prover a plena saúde a todos. 
b) Formação médica no Brasil: os médicos brasileiros são formados, quase em sua totalidade, para um exercício profissional liberal, muito distante das necessidades sanitárias da população, uma vez que o exercício da medicina no consultório privado é muito diferente do exercício na saúde pública. Assim, há um verdadeiro paradoxo entre a formação e o exercício profissional, uma vez que a maior parte dos médicos trabalha no SUS, mesmo que parcialmente. Além disso, a formação médica atual, voltada para medicina de mercado aquela ligada predominantemente à procedimentos -, deixa lacunas no conhecimento técnico, pois não prepara o profissional para uma visão mais crítica e integral da medicina voltada para saúde pública - ligada aos processos que envolvem a saúde, tornando o profissional, dessa forma, uma presa fácil para uma medicina de mercado, que impõe prescrições de medicamentos e solicitações de exames de maneira acrítica.

c) Concepção de que as políticas públicas são ruins ou os investimentos, insuficientes: é frequente o uso desse lugar comum para justificar a judicialização e formar 0 convencimento dos magistrados. No entanto, quando analisadas as demandas judiciais, percebe-se que os temas são recorrentes: medicamentos de alto custo, prótese importadas, medicamentos de marca, obstinação terapêutica, desconhecimento das políticas públicas, entre outros (6). É importante ressaltar que o país tem um importante arsenal normativo sobre padronização de medicamentos essenciais e especializados, rol de procedimentos tanto do poder público como da saúde suplementar, diversas diretrizes e protocolos em todos os níveis de atenção. No entanto, pouco consultados ou conhecidos.

d) A ideia de que a Medicina é um bem de mercado e a Saúde um bem de consumo: os médicos com baixa qualificação técnica, atrelada à pressão feita pelos laboratórios farmacêutico e pelas indústrias de equipamentos, levam a distorções, práticas equivocadas e desvios éticos, associado a um acesso ilimitado a informações leigas e direcionadas ao consumo. No limite, tratam-se como direitos, tecnologias que não melhoram nem curam, e que, na maior parte das vezes aumentam o sofrimento e reduzem a vida do paciente (7).

e) Omissão do julgador: O Judiciário parte da falácia da inquestionabilidade da prescrição médica, pois o julgador não possui bases técnicas para definir se o pleito é devido e está baseado em evidências. Ao final, tem-se a imposição de um poder (o médico) a outro poder (o Executivo) intermediada pelo Judiciário, de modo que o Poder Judiciário passa a ser um meio operacional de incorporação - acrítica- de tecnologias e da disseminação do 
seu uso em escala comercial, muitas vezes medicamentos não essenciais ou não garantidos em termos de eficácia e segurança (7).

Ações mais recentes do Ministério da Saúde (MS) e do Conselho Nacional de Justiça (CNJ), assim como iniciativas pontuais e cada vez mais frequentes de membros do Poder Judiciário estão questionando este modus operandi, influenciando a interpretação do artigo 196 para "a saúde é direito de todos e dever do Estado, garantido mediante políticas sociais e econômicas, além de afirmar que o SUS brasileiro se funda na Medicina baseada em evidências (art. 19-Q, § 2 $\mathrm{n}^{\circ} \mathrm{I}$. da Lei 8.080/90, com a redação dada pela Lei 12.401/11)", sendo este, "minimamente, o ponto de equilíbrio entre a efetividade do direito à saúde e a chamada cláusula da reserva do possível” (8), sendo a tecnologia efetiva. Se não for, não há direito à saúde no caso.

Ocorre que as políticas públicas possuem algumas falhas ao aplicar, no caso concreto, os princípios do SUS, e a judicialização deve ser vista como um instrumento excepcional, não como regra do sistema. As principais medidas adotadas foram o uso de evidência científica na tomada de decisão dos Poderes Executivo e Judiciário e a preocupação com a sustentabilidade do financiamento da saúde.

\section{Histórico da Avaliação de Tecnologias em Saúde evolução dos gastos em sentenças judiciais na saúde (ATS) no Ministério da Saúde (MS)}

De acordo com informações do site do MS (9), a estruturação da área de ATS foi iniciada com a criação do Conselho de Ciência, Tecnologia e Inovação em Saúde (CCTI), em 2003. O Conselho é responsável pela condução de diretrizes e pela promoção da avaliação tecnológica para incorporação de novos produtos e processos pelos gestores, prestadores e profissionais dos serviços no SUS.

Dentro de sua estrutura, o Departamento de Ciência e Tecnologia (Decit) criou, em 2005, uma área voltada para a ATS, para consolidar o trabalho desenvolvido pelo grupo técnico (GT/ATS), com os seguintes propósitos: promover estudos de ATS para subsidiar a tomada de decisão no SUS, monitorar a utilização de tecnologias já incorporadas ao SUS, capacitar os gestores e profissionais de saúde e disseminar resultados da ATS para os gestores. 
Se entende por tecnologias em saúde: medicamentos, dispositivos médicos, procedimentos, sistemas organizacionais e de suporte dentro dos quais os cuidados à saúde são oferecidos e exames diagnósticos.

Lançada em 2008, a Rede Brasileira de Avaliação de Tecnologias em Saúde (Rebrats) tem por princípio o estabelecimento de elos entre pesquisa, política, gestão e pesquisa. Coordenada pelo Decit, a rede reúne instituições gestoras do SUS, instituições de ensino e pesquisa, unidades de saúde, hospitais, sociedades profissionais e de usuários.

Alguns resultados das atividades desenvolvidas pelos grupos de trabalho da rede foram: a implantação de NATS em hospitais públicos de ensino.

Os NATS em Hospitais de Ensino são compostos, inicialmente, por 24 instituições de todas as regiões do País.

Os NATS buscam introduzir a cultura de ATS nesses hospitais, por meio da utilização de evidências disponíveis para auxiliar o gestor hospitalar a tomar decisões quanto à inclusão de novas tecnologias, à avaliação de tecnologias difundidas e ao uso racional.

São atribuições dos NATS:

I - promover capacidade técnica para a inserção de instituições na Rede Nacional de Avaliação de Tecnologias em Saúde (REBRATS);

II - desenvolver ações para a capacitação permanente de profissionais e técnicos;

III - incentivar e produzir pesquisas, estudos e revisões sistemáticas voltadas ao uso da evidência científica na tomada de decisão;

IV - coordenar a revisão de diretrizes clínicas dos hospitais, em consonância com as necessidades do SUS;

V - incentivar e capacitar tutores para orientar alunos e profissionais de saúde para executar atividades de ensino e pesquisa voltados para avaliação de tecnologias em saúde;

VI - sensibilizar e incentivar os profissionais dos hospitais à introdução da cultura de avaliação de tecnologias em saúde; e

VII - fomentar a articulação entre ensino e serviço na área de avaliação de tecnologias em saúde e saúde baseada em evidências. (10) 


\section{As origens do NATS HC-UFMG}

O HATS HC/UFMG foi fundado em 2008 como Órgão Assessor da Diretoria do Hospital das Clínicas e referendado pelo Reitor da UFMG em 2009. Em 2009, atendendo a edital do MS foi reconhecido como Centro de Avaliação de Tecnologias em Saúde passando a fazer parte da REBRATS.

O HC/UFMG é um hospital de ensino, público e federal, que tem a missão de "desenvolver a assistência na área da saúde, com eficácia e eficiência e, de forma indissociável e integrada, o ensino, a pesquisa e a extensão, constituindo-se como referência junto à sociedade" (11).

O NATS fornece análises críticas sobre novas tecnologias e processos, seu valor clínico e suas possibilidades de incorporação na prática clínica, além de promover a prática em saúde baseada em evidências, por meio de núcleos de estudos, prática e disseminação do conhecimento, assim como a cultura dos ciclos de melhoria pelo aprendizado e liderança compartilhados.

Oferece uma gama de serviços como projeto de extensão, particularmente instituições governamentais e empresas do ramo de saúde. O NATS HC/UFMG desenvolve projetos e parcerias com MS (DECIT, CONITEC), Agencia Nacional de Vigilância de Saúde (ANVISA), Programa de Desenvolvimento das Nações Unidas (PNUD), Organização Pan-Americana de Saúde (OPAS) e IATS (Instituto de Avaliação de Tecnologias em Saúde). 


\section{Procedimento de Elaboração da Nota Técnica}

As NT elaboradas seguem o seguinte fluxo:

Figura 1 - Fluxo na solicitação/elaboração da NT pelo NATS HC-UFMG

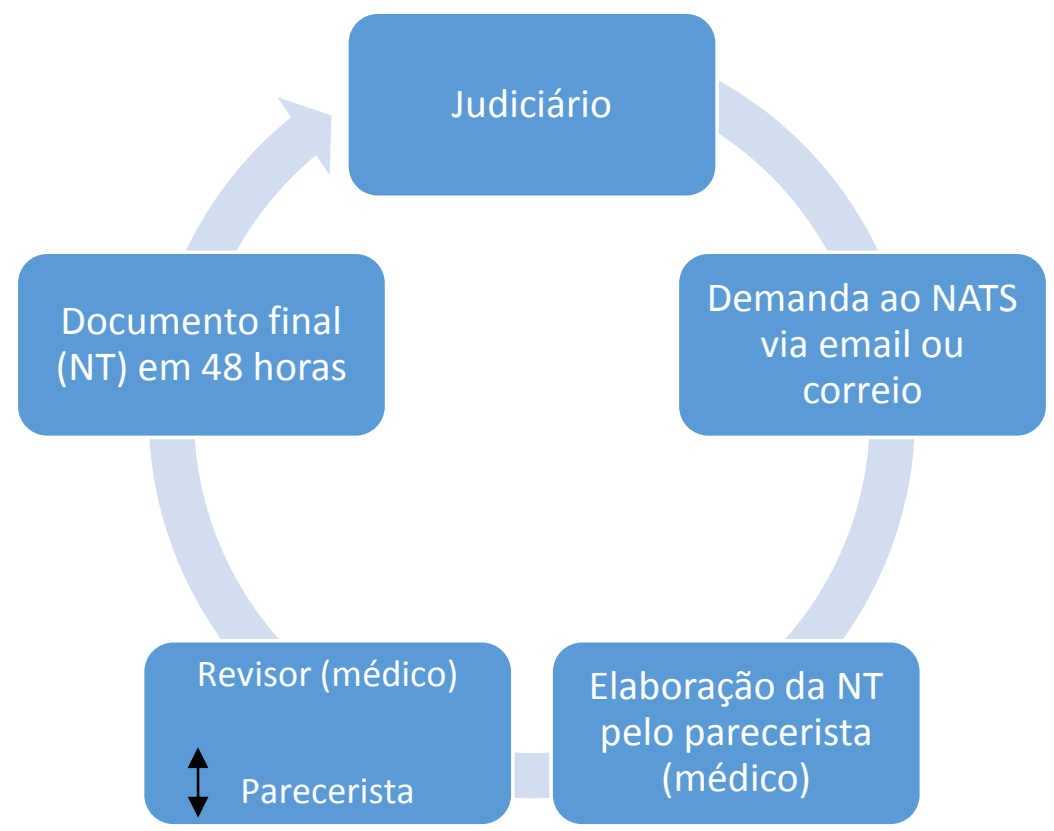

\section{Caracterização da Nota Técnica}

Os avanços científicos da Medicina nos últimos anos, as facilidades crescentes nas tecnologias de informação e comunicação significam grandes desafios para os profissionais de saúde. Sobrecarregados com tanta informação, com frequência se veem confundidos por propagandas enganosas de efeitos benéficos exagerados de medicamentos e procedimentos. Para tal, a medicina baseada em evidência (MBE) procura integrar a experiência clínica com a melhor evidência externa disponível oriunda da pesquisa sistemática da literatura científica. Para confecção da NT para o Poder Judiciário são utilizados os princípios da MBE descritos de forma resumida a seguir:

\section{Estrutura mínima da NT:}

a) Solicitação: reproduz a demanda do judiciário e os relatórios dos profissionais de saúde sobre a demanda. 
b) Descritores de ciência em saúde (DeCS): foram criados pela Biblioteca Virtual em Saúde (BIREME) para uso na indexação de artigos de revistas científicas, livros, anais de congressos, relatórios técnicos, e outros tipos de materiais, assim como para ser usado na pesquisa e recuperação de assuntos da literatura científica nas bases de dados LILACS, MEDLINE e outras.

c) Contexto clínico: resumo sobre a doença em questão, com dados epidemiológicos.

d) Descrição da tecnologia a ser avaliada: especifica os detalhes da tecnologia solicitada (seja medicamento, produto para saúde, procedimentos em saúde, exames complementares, etc)

e) Pergunta estruturada: P.I.C.O. representa um acrônimo para Paciente, Intervenção, Comparação e "Outcomes" (desfecho). Esses quatro componentes são os elementos fundamentais da questão de pesquisa e da construção da pergunta para a busca bibliográfica de evidências.

f) Estratégia de busca: realizada nas bases de dados científicas, de acordo com a pergunta estruturada. Deve ser passível de ser reproduzida. A partir dessa busca serão selecionados os estudos científicos que irão "responder" à pergunta P.I.C.O.

g) Resultado da revisão da literatura: São apresentados os estudos selecionados (a melhor evidência científica disponível, ou não, sobre o tema em questão). Deverá ser realizada uma avaliação crítica da literatura disponibilizada com relação ao (s) tipo (s) de estudo, se metodologia científica foi adequada. O ponto final será definir se baseado nos estudos encontrados, se a evidência científica é forte, fraca ou mesmo inexistente, sobre a tecnologia em questão.

h) Disponibilidade ou opções terapêuticas no SUS: Informar se a tecnologia é disponibilizada pelo SUS ou não.

i) Considerações: Discussão sobre os estudos encontrados na literatura científica e correlacionar as evidências científicas com a tecnologia demandada.

j. Recomendação - Recomendação técnica final do NATS, a favor ou contra a tecnologia solicitada para o caso clínico em questão.

As NT nem sempre incluem o preço da tecnologia. Se for medicamento, é consultada a lista CMED, mas se for produto para saúde, geralmente faz-se busca manual no google. 


\section{Resultados}

Desde o início do convênio já foram elaboradas cerca de 2.000 notas técnicas, sendo que 1600 foram elaboradas em processos em tramitação na primeira instância e 400 em processos em tramitação na segunda instância, sendo atendidas 114 comarcas.

O Poder Judiciário de Minas Gerais já se manifestou em fóruns e congressos acerca da importância das informações técnicas, prestadas pelas NT, para a tomadas de decisões. As NT fornecidas pelo NATS se mostram de grande valia, porque permitem aos magistrados decidir a partir de informações sobre a evidência científica daquilo que estava sendo apreciado. Inclusive, a liderança da Desembargadora aposentada do TJMG Vanessa Verdolim Hudson Andrade e do desembargador H Renato Dresch, também do TJMG, ferrenho defensor do uso da MBE nos processos que envolvem a judicialização, foi e continua sendo indispensável para o sucesso do trabalho do NATS e para a aceitação do mesmo junto aos magistrados.

Com a medida, busca-se evitar a prolação de decisões judiciais muitas vezes destituídas da boa técnica médica, com benefícios para todos, o que resulta, também, no uso mais racional e adequado dos recursos públicos.

Dois resultados imediatos advieram deste convênio: Primeiro, a mudança de entendimento dos membros do Poder Judiciário, com membros defendendo a tese da Justiça Baseada em Evidências. E em segundo, a surpreendente reversão de gastos do Estado de Minas Gerais com as ações judicializadas: (12) 
Figura 2 - Evolução dos gastos com sentenças Judiciais na Saúde

\section{Evolução dos Gastos em Sentenças} Judiciais na Saúde

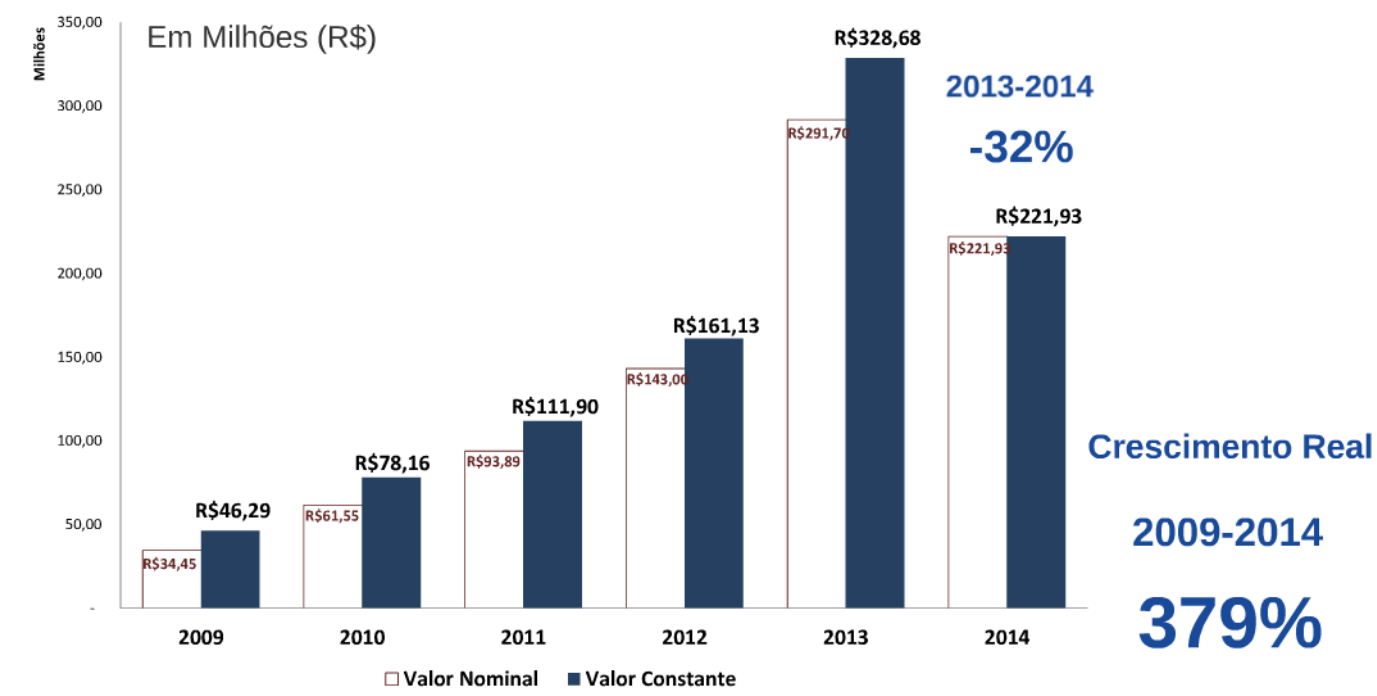

Enṇte. Armazém de Infnrmarñoc SIAC!

\section{Desafios}

a) Financiamento

O convênio entre NATS HC-UFMG, SES e TJMG foi realizado entre 2012-2014, portanto, no momento em que esse artigo é escrito, agosto de 2016, o referido convênio não está em vigor. Isso porque entraves burocráticos impediram que a Procuradoria do Estado conseguisse viabilizar a continuidade do convênio, uma vez que há um novo entendimento por parte da Procuradoria no qual a contratação com a Fundação de Ensino e Pesquisa da UFMG (FUNDEP-UFMG) só é possível mediante prévia licitação. Pelo exposto, atualmente - NATS só tem elaborado NT para a saúde suplementar, por meio de um termo de cooperação firmada entre o TJMG e o Instituto Brasileiro para Estudo e Desenvolvimento do Setor de Saúde (IBEDESS), desde 2014 e que já possibilitou a elaboração de 160 NT em processos judiciais em tramitação em 12 comarcas de Minas Gerais. Portanto, é necessário um arranjo institucional que dê garantias de continuidade para atuação do NATS. 
No dia 23 de agosto de 2016 foi assinado um termo de cooperação entre o MS e o CNJ (13) para possibilitar acesso dos magistrados a pareceres e estudos clínicos de MBE. Contudo, ainda não está claro como esse termo afetará o trabalho do NATS.

b) Convencimento do Magistrado

É fundamental para o Poder Judiciário e, especialmente para a manutenção da discricionariedade do juiz que a instituição que elabora as Notas Técnicas seja absolutamente isenta de conflitos, além de ser referência técnica para a sociedade. $\mathrm{E}$, com estas características, o NATS HC-UFMG se apresenta como opção em Minas, o que se insere nas hipóteses de dispensa de licitação conforme art. 24, XIII (14), da lei federal 8.666/93.

O diferencial do serviço prestado pelo NATS para o Poder Judiciário tem como base análise essencialmente técnica, baseada na melhor evidência científica disponível, sobre a tecnologia em questão.

c) Aproximação com o Poder Judiciário

Com o objetivo de conscientizar os magistrados da importância do trabalho desenvolvido, o NATS participa de todos os fóruns que é convidado, pois nesses eventos é possível expor o trabalho e a metodologia desenvolvidos, sendo palpável o aumento exponencial na demanda e o maior entendimento da importância em seguir as conclusões das NT com essas participações. Todavia ainda existem desafios, como os apresentados abaixo:

Transcrever os termos médicos técnicos de forma a fornecer clareza e compreensão para os magistrados, sem comprometer a evidência científica é um desafio a ser aprimorado sempre, especialmente porque o NATS HC-UFMG nunca teve verba para contratar profissionais ou estagiários do Direito para auxiliá-los na elaboração das NT.

Outra dificuldade encontrada é o tempo exíguo para resposta, pois os magistrados concediam entre 48 e 72 horas para a elaboração da NT. Isso porque muitas vezes, dependendo do grau de dificuldade do assunto, a busca pela melhor evidência dentro das bases de dados científicas disponíveis, a leitura crítica dessa literatura encontrada e a transcrição em forma de NT dentro de uma linguagem acessível é também um constante exercício de aperfeiçoamento para os profissionais. Além disso, a nota técnica, sempre passa pela revisão de outro médico, que tem o importante papel, não só de avaliar o documento do ponto de vista técnico, como também de levantar e apontar dúvidas à respeito 
da clareza daquilo que foi explicado tecnicamente. Essa revisão muitas vezes não acontece uma única vez, até que o documento final seja liberado. E, com o tempo exíguo, muitas vezes não é possível se ater a todos os detalhes.

A falta de acesso ao resultado final da demanda também é um desafio para o convênio, pois os profissionais que emitem as NT têm interesse em saber se as NT produzidas são relevantes para o deslinde da demanda, até para aperfeiçoarem seu trabalho. Contudo, como o NATS não conta com profissional do direito apoiando, não consegue ter acesso às decisões, seja porque os profissionais não compreendem a linguagem esposada nas sentenças e acórdãos, quer porque não possuem condições de irem até as comarcas do interior para extraírem cópias das decisões.

d) Participação dos gestores

A participação dos gestores é vital para aprimoramento dos processos e para a efetividade do trabalho desenvolvido pelos NATS. Isso porque um gestor comprometido consegue analisar e usar os resultados do trabalho do NATS como um observatório de problemas que devem ser enfrentados e, assim, contribuir para a diminuição da judicialização e melhorar seus próprios processos.

A título de exemplo, ficam aqui duas sugestões para atuações dos gestores:

- Registro e monitoramento dos pacientes dos casos judicializados e deferidos mais frequentes e onerosos (como por exemplo, medicamentos oncológicos e produtos para saúde utilizados em procedimentos cirúrgicos)

- Organizar um ambulatório para os pacientes com degeneração macular relacionada com a idade (DMRI) que tiveram seus pedidos deferidos a favor do tratamento com injeção intra-vítrea de medicação antiangiogênica, com a finalidade de verificar se permanecem as condições clínicas mínimas para aplicação da terapia. Embora haja evidência do tratamento, muitos pacientes já perderam a oportunidade de obter resultados com a terapia no momento da aplicação.

e) Fóruns de discussão com a sociedade médica

A aproximação com os médicos prescritores é um grande desafio da parceria, pois é necessária a conscientização destes profissionais acerca do impacto social e econômico que a prescrição sem evidência científica provoca. Essa conscientização precisa ser feita por meio de fóruns de discussão com a sociedade médica e, enquanto não for feita os 
processos que envolvem judicialização da saúde continuarão necessitam da emissão de NT com a finalidade de demonstrar a posição da MBE sobre a prescrição médica.

\section{Considerações finais}

Percebe-se com o caso concreto apresentado que o NATS pode ajudar a diminuir o custo do Poder Público com a judicialização da saúde. Atualmente, essa ajuda se dá com a emissão de NT, com o objetivo de amparar as decisões judiciais, contudo, emitir NT eternamente não é solução para o problema da judicialização da saúde e nem figura nos objetivos do NATS HC-UFMG.

O que o NATS HC-UFMG percebeu nesses anos de convênio com o TJMG é que não há solução única e sim a necessidade de contínuo processo de integração entre os atores que compõe a judicialização da saúde: gestores, médicos prescritores, magistrados, universidades e pacientes e o NATS pode ser ator neutro para mediar essa integração.

\section{Referências}

1. Núcleo de Avaliação de Tecnologias em Saúde da Universidade Federal de Minas Gerais. Nota Técnica sobre sobre a indicação dos medicamentos trastuzumabe e pertuzumabe para tratamento de adenocarcinoma de cólon metastático, de 09 de setembro de 2015.

2. Conselho Nacional de Justiça. Recomendação 31 de 31 de março de 2010. Disponível em: $\quad<$ http://www.cnj.jus.br/files/atos_administrativos/recomendao-n31-30-03-2010presidncia.pdf> Acesso em 20 ago. 2016.

3. Lopes, A. A. Medicina Baseada em Evidências: a arte de aplicar o conhecimento científico na prática clínica. RevAssMed Brasil 2000; 46(3), p. 285-8.

4. Brasil. Constituição da República Federativa do Brasil. Disponível em: http://www.planalto.gov.br/ccivil_03/Constituicao/Constituicao.htm Acesso em 20 ago. 2016.

5. Teixeira, C. Os princípios do Sistema Único de Saúde. Disponível em: http://www.saude.ba.gov.br/pdf/OS_PRINCIPIOS_DO_SUS.pdf Acesso em 22 ago. 2016.

6. Araújo, C P. A judicialização da saúde e a necessidade de assessoramento técnico do Juiz para decidir. Disponível em: http://www.comitesaudemg.com.br/www/wpcontent/uploads/2016/03/Artigo-livro-Rodrigo-vers\%C3\%A3o-final.pdf Acesso em 25 ago. 2016. 
7. Gadelha, MIP. O papel dos médicos na judicialização da saúde. Revista CEJ, Brasília, Ano XVIII, n. 62, p. 65-70, jan./abril 2014.

8. Justiça Federal de Minas Gerais. Ação Ordinária n. 79631-83.2014.4.01.3800. Juiz de Direito Marcelo Dolzany da Costa. 16ํㅡ Vara da Federal. DJ: 17.04.2015.

9. Ministério da Saúde. Ciência, Tecnologia e Inovação em Saúde. Disponível em: http://bvsms.saude.gov.br/bvs/folder/folder\%20institucional.pdf. Acesso em 22 ago. 2016.

10. Rede Brasileira de Avaliação de Tecnologias em Saúde. NATS. Disponível em: http://rebrats.saude.gov.br/institucional/nats. Acesso em 22 ago. 2016.

11. Hospital das Clínicas da Universidade Federal de Minas Gerais. Missão, visão e valores. Disponível em <http://www.ebserh.gov.br/web/hc-ufmg/missao-visao-e-valores>, Acesso em 23 ago. 2016.

12. Tribunal de Constas do Estado de Minas Gerais. Impacto Orçamentário da judicialização da saúde no SUS. Disponível em www.tce.mg.gov.br Acesso em 24 ago. 2016.

13. Ministério da Saúde. Termo de cooperação vai dar apoio técnico nas ações da saúde. Disponível em: < http://portalsaude.saude.gov.br/index.php/cidadao/principal/agenciasaude/25231-termo-de-cooperacao-vai-dar-apoio-tecnico-nas-acoes-da-saude>, acesso em 24 ago. 2016.

14. Brasil. Lei 8.666 de 21 de junho de 1993. Disponível em: < http://www.planalto.gov.br/ccivil_03/leis/L8666cons.htm>. Acesso em 23 ago. 2016.

\section{Como citar este artigo:}

Nogueira JL, Carvalho L, Dadalto L. Parcerias entre Universidades e Poder Judiciário: experiência de Minas Gerais. Revista Cadernos Ibero-Americanos de Direito Sanitário. 2017 jan./mar, 6(1): 55-71. 